\title{
Cognitive Deficits in Rats after Forebrain Cholinergic Depletion are Reversed by a Novel NO Mimetic Nitrate Ester
}

\author{
Brian M Bennett*,', James N Reynolds', Glen T Prusky², Robert M Douglas ${ }^{3}$, Robert J Sutherland ${ }^{2}$ and \\ Gregory RJ Thatcher ${ }^{4}$
}

'Department of Pharmacology \& Toxicology, Queen's University, Kingston, ON, Canada; ${ }^{2}$ Canadian Centre for Behavioural Neuroscience, The University of Lethbridge, Lethbridge, AB, Canada; ${ }^{3}$ Department of Ophthalmology, University of British Columbia, Vancouver, BC, Canada;

${ }^{4}$ Department of Medicinal Chemistry \& Pharmacognosy, College of Pharmacy, University of Illinois at Chicago, Chicago, IL, USA

\begin{abstract}
Many conditions adversely affecting learning, memory, and cognition are associated with reductions in forebrain acetylcholine (ACh), most notably aging and Alzheimer's disease. In the current study, we demonstrate that bilateral depletion of neocortical and hippocampal ACh in rats produces deficits in a spatial learning task and in a recently described, delayed visual matching-to-sample task. Oral administration of the novel nitrate, GTI06I (4-methyl-5-(2-nitroxyethyl) thiazole HCl), and the acetylcholinesterase inhibitor, donepezil, reversed the cognitive deficits in both memory tasks in a dose-dependent manner. GTI06I was superior in the delayed matching-tosample task. GTI06I was absorbed rapidly after oral administration, crossed the blood brain barrier, and achieved brain concentrations that were slightly higher than those found in plasma. The activity of GTI06I was NO mimetic: soluble guanylyl cyclase (sGC) was activated, but selectivity was observed for $\mathrm{SGC}$ in the hippocampus relative to the vasculature; and hippocampal levels of phosphorylated ERKI/2, which is a postulated intermediary in the formation of long-term memory, were increased. The beneficial effect on visual and spatial memory task performance supports the concept that stimulating the NO/sGC/cGMP signal transduction system can provide new, effective treatments for cognitive disorders. This approach may be superior to that of current drugs that attempt only to salvage the residual function of damaged cholinergic neurons.

Neuropsychopharmacology (2007) 32, 505-513. doi: I0.1038/sj.npp. I 30 I054; published online 8 March 2006
\end{abstract}

Keywords: nitric oxide; Alzheimer's disease; ERK; cGMP; cognition; memory

\section{INTRODUCTION}

Alzheimer's disease (AD) is characterized by disruption of both excitatory amino acid and cholinergic neurotransmission. Loss of cholinergic neurons, and subsequent deficits in cholinergic neurotransmission in the hippocampus and cerebral cortex, is strongly correlated with clinical signs of cognitive impairment and dementia in $\mathrm{AD}$ patients (Mesulam, 2004). The use of acetylcholinesterase inhibitor drugs, such as donepezil (Aricept), is based upon the cholinergic hypothesis of memory loss and cognitive deficit (Bartus et al, 1982). These drugs have represented the sole therapeutic response to $\mathrm{AD}$ until the recent introduction of the $N$-methyl-D-aspartate (NMDA) receptor low affinity antagonist, memantine. The clinical efficacy of acetylcholinesterase inhibitors has been questioned (Courtney et al,

*Correspondence: Dr BM Bennett, Department of Pharmacology \& Toxicology, Queen's University, Kingston, ON, Canada K7L 3N6, Tel: + 613533 6473, Fax: + 613533 6412,

E-mails: brian.bennett@queensu.ca; thatcher@uic.edu

Received 27 December 2005; revised 17 January 2006; accepted 18 January 2006

Online publication: 30 January 2006 at http://www.acnp.org/citations/ Npp0 I 3006050770/default.pdf
2004), and memantine is not approved for mild to moderate forms of $\mathrm{AD}$ (Areosa et al, 2005), thus the imperative for new therapeutic agents to treat $\mathrm{AD}$ and other dementias remains.

Signal transduction by the NO/soluble guanylyl cyclase (sGC)/cGMP system modulates synaptic transmission and plasticity in the CNS, and in vivo studies suggest an important role for NO/cGMP in the acquisition of new learning and memory (Bernabeu et al, 1997; Prickaerts et al, 2002; Prickaerts et al, 1997). This is supported by numerous in vitro studies indicating a role in long-term potentiation (LTP), clustering of presynaptic and postsynaptic proteins (suggesting a role in synaptogenesis), and enhancement of presynaptic neurotransmitter release. Aberrant NO signaling in the brains of $\mathrm{AD}$ patients has also been reported (Bonkale et al, 1995). Thus, sGC activation and cGMP formation in the brain may be an effective strategy for mitigating the cognitive dysfunction that occurs as a consequence of cholinergic deficits in the CNS. In contrast to the acetylcholinesterase inhibitors that attempt to augment the residual function of a degenerating cholinergic system, NO mimetics are postulated to bypass this system and modulate the function of signaling pathways downstream from cholinergic receptor activation. 
Most recently, two studies have directly implicated a role for dysfunction of NO/cGMP signaling in AD. In hippocampal slices, disruption of LTP by amyloid- $\beta$ peptide $(\mathrm{A} \beta)$ was shown to result from a malfunction of NO/cGMP signaling (Puzzo et al, 2005). Further, Snyder et al (2005) reported that $\mathrm{A} \beta$ produced a depression of NMDA-evoked currents via endocytosis of NMDA receptors, mediated via dissociation of the synaptic protein, PSD95, from the NMDA receptor. As both $\mathrm{SGC}$ and neuronal nitric oxide synthase (NOS) are known to be associated with PSD95 in the brain (Russwurm et al, 2001), this work suggests that $\mathrm{A} \beta$ specifically disrupts hippocampal NO/cGMP signaling. It was concluded that intervention with NO donors or drugs enhancing NO/cGMP signaling might provide a novel approach to treatment of AD (Puzzo et al, 2005).

Classical nitrates, of which glyceryl trinitrate (GTN) is the archetype, are NO mimetics, in that they manifest many aspects of the biological activity of $\mathrm{NO}$ in vivo (Bennett et al, 1994; Thatcher et al, 2004). Although the hypotensive effect of GTN itself precludes its use in CNS-directed therapy, we have previously reported a novel nitrate that demonstrates: (a) attenuated hypotensive activity relative to GTN; (b) neuroprotection in the middle cerebral artery occlusion model of ischemic stroke; and (c), reversal of scopolamineinduced cognition deficits in rats tested in the Morris water task (MWT) (Reynolds et al, 2002; Smith et al, 2000).

Prusky et al (2004) recently demonstrated long-delay trial-unique visual matching-to-sample (DMTS) performance in rats, implicating essential roles for both the hippocampus and perirhinal cortex. Thus, the hippocampus is important not only for spatial memory, but also for visual recognition memory, a cognitive process that is disrupted in $\mathrm{AD}$ (Lindeboom et al, 2002). In this paper, we use this DMTS task to examine the reversal of a cholinergic deficit in recognition memory by a novel nitrate, GT1061 (4-methyl5-(2-nitroxyethyl) thiazole $\mathrm{HCl}$ ), and compare both to effects on spatial working memory and to the effects of donepezil in the same tasks. A potential mechanism of action of this novel compound in the hippocampus also is described.

\section{MATERIALS AND METHODS}

All procedures for animal experimentation were undertaken in accordance with the principles and guidelines of Canadian Council on Animal Care.

\section{Behavioral Studies}

Moving hidden platform Morris water task: surgery. In these studies, 90 of 100 young adult female Long-Evans rats received bilateral injections of 192 IgG-saporin into the nucleus basalis magnocellularis and medial septum to deplete the cholinergic innervation of the cerebral cortex and hippocampus, respectively (Pizzo et al, 1999). Rats were anaesthetized with isoflurane and under stereotaxic guidance (coordinates: medial septum; AP + 0.6, L 1.2, D -7.5, -6.8 from bregma; nucleus basalis; AP $-1, \mathrm{~L} \pm 3, \mathrm{D}-7.5$ ), 192 IgG-saporin $(0.1 \mu \mathrm{g}$ in $1.0 \mu \mathrm{l}$ of phosphate-buffered saline, $\mathrm{pH} 7.4$, over $15 \mathrm{~s}$ ) was infused into each site. The remaining 10 animals received sham surgery. All rats were allowed to recover for 14 days.
MWT testing. In the moving hidden platform version of the MWT, the platform was moved to a new location within the pool every other day according to a random sequence of locations. Thus, for two consecutive days the platform remained in the same location. On the third day, the platform was moved to a new location for another pair of days, and this was continued until six locations had been completed. Rats received eight trials each day. Data were collected using a video camera and microcomputer automated system (HVS 2020 Plus system). On every trial, time and distance to find the platform was automatically recorded and analyzed. Following recovery from surgery, the rats were divided into 10 groups; sham operated vehicle control $(n=10)$, lesion vehicle control $(n=10)$, four groups, each receiving one dose of donepezil $(0.05,0.5$, 0.1 , and $1 \mathrm{mg} / \mathrm{kg})(n=10)$, and four groups, each receiving one dose of GT1061 $(0.5,1,5$, and $10 \mathrm{mg} / \mathrm{kg})(n=10)$. Each rat received the appropriate drug by gavage $60 \mathrm{~min}$ before testing each day. Each day the rats were tested in the MWT. Following the last day of testing, the animals were perfused with $4 \%$ paraformaldehyde and their brains removed, cryoprotected, and sectioned for acetylcholinesterase staining (Karnovsky and Roots, 1964).

Delayed matching-to-sample task. The testing apparatus and DMTS training and testing procedures are detailed in a previous publication (Prusky et al, 2004). Briefly, adult female Long-Evans rats $(n=10)$ were first trained on a simple visual discrimination task, and then in four stages, were gradually shifted to the trial-unique, one-trial learning task. Throughout the testing, rats were rewarded for identifying and swimming toward a correct picture where they could escape from water to a submerged platform. The black-and-white pictures were displayed on computer monitors to eliminate nonvisual cues. The pictures were drawn from a set of more than 100 and on any given day no picture appeared on more than one trial, such that, relative familiarity of pictures is irrelevant. Similarly, the probability of any specific picture being used as a sample or a foil was equal. In the final stage, during each trial, rats viewed a picture in a sample phase, and then in a choice phase, discriminated the sample picture from a novel picture with high accuracy in a different pool located in the same room. The left/right position of the correct picture varied randomly to make the task nonspatial. The delay dependence of memory for the sample picture was measured systematically by varying the delay between the sample and choice phases. All animals received approximately 6 weeks of presurgical training. At 1-week after surgery, DMTS performance was remeasured. On the basis of the presurgical memory performance we selected a 4-min interval for postsurgical testing of drug effects. Presurgical performance at this delay interval between sample and choice was well-above chance for each rat, but was less than maximum accuracy (Figure 1). Thus, it afforded us the opportunity to measure deficits, drug effects, and dose response, at a moderate level of memory difficulty (thereby avoiding floor or ceiling effects). Performance in the DMTS task before and after surgery was compared at the 4-min interval. Drug or drug vehicle was administered by gavage 30 -min before testing. Each rat was tested with vehicle solution and three doses each of GT1061 and donepezil, thus, the experimental 


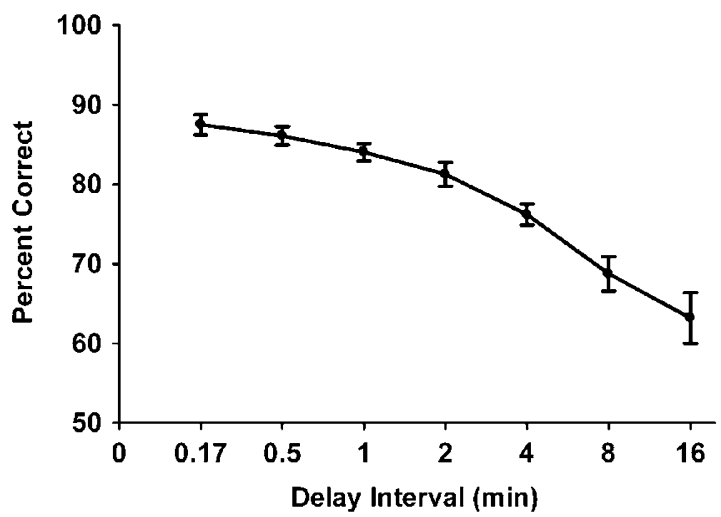

Figure I Mean preoperative visual matching-to-sample performance at each training delay interval. Choice accuracy was assessed in each animal during 24 trials at each interval, and declined systematically with increasing delays. Data are expressed as the mean \pm SEM $(n=7)$.

design was fully 'within subjects'. The doses of GT1061 were 1,5 , and $10 \mathrm{mg} / \mathrm{kg}$ and for donepezil were $0.1,0.5$, and $1.0 \mathrm{mg} / \mathrm{kg}$. There was at least a 48 -h time interval between successive tests of drugs or vehicle. The order of testing was always vehicle, GT1061 5, 10, and $1 \mathrm{mg} / \mathrm{kg}$ and then donepezil 0.5, 1.0, and $0.1 \mathrm{mg} / \mathrm{kg}$. Between GT1061 and donepezil testing we retested vehicle administration, and found that performance was not different from that observed with the first vehicle test $(55.0 \pm 2.2 \%$ correct $v s$ $57.3 \pm 1.9 \%$ correct, $p=0.35$, Student's $t$-test for paired data). On each day of testing, each rat received 12 memory trials and the percentage correct choices was calculated. In a separate experiment, we assessed whether GT1061 enhanced cognitive performance in nonlesioned animals. Rats $(n=4)$ were trained as described above and DMTS performance evaluated after oral administration of $10 \mathrm{mg} / \mathrm{kg}$ GT1061. This was compared to DMTS performance assessed on the day before and the day subsequent to drug administration.

Surgery involving DMTS rats. After initial training, the cholinergic innervation of the hippocampus and cerebral cortex was depleted by intraventricular microinjection of 192-IgG saporin $(1.8 \mu \mathrm{g}$ in $5 \mu \mathrm{l}$ of phosphate-buffered saline, $\mathrm{pH} 7.4$, over $15 \mathrm{~s}$ ) into the lateral ventricle of each cerebral hemisphere (coordinates: AP $-1.1, \mathrm{~L} 1.5, \mathrm{D}-4.2$ from bregma).

Pharmacokinetic studies. Male Sprague-Dawley rats (250$275 \mathrm{~g}$ ) were fasted overnight, anaesthetized for surgery with a combination of Hypnorm/Versed and then catheters (30gauge Teflon fused to 0.02 -inch inside diameter Tygon ${ }^{\circledR}$ ) were inserted into the abdominal aorta and abdominal vena cava for blood sampling and drug administration, respectively. Animals were administered $50 \mu \mathrm{mol} / \mathrm{kg}(11.2 \mathrm{mg} / \mathrm{kg})$ GT1061 (prepared in saline) by the i.v., i.p., or oral routes and blood samples $(250 \mu \mathrm{l})$ obtained from the aortic catheter at various times. Blood was immediately centrifuged and $100 \mu \mathrm{l}$ aliquots of plasma were assayed for GT1061 by gas chromatography with electron capture detection. The data were fitted to a 2-compartment open model using PKSolutions software (Summit Research Services, Montrose, CO) to derive pharmacokinetic parameters. To assess the distribution of GT1061 into the CNS, rats were fasted overnight and then administered $25 \mu \mathrm{mol} / \mathrm{kg}(5.6 \mathrm{mg} / \mathrm{kg})$ GT1061 by gavage. Five minutes later the animals were killed and a blood sample was obtained. The brain was removed and one hippocampus dissected out and frozen in liquid nitrogen, procedures lasting approximately $5 \mathrm{~min}$ to complete. The hippocampus was weighed, pulverized, and then both plasma and hippocampus samples assayed for GT1061 by gas chromatography. To assess potential sex and strain differences in the pharmacokinetic properties of GT1061, female Long-Evans rats were instrumented as described above, and blood levels of GT1061 determined after oral administration of $50 \mu \mathrm{mol} / \mathrm{kg}$ GT1061. The area under the plasma concentrationtime curve from 0 to $120 \mathrm{~min}$ was $82.1 \pm 36.2 \mu \mathrm{mol}-\mathrm{min} / 1$ (SEM, $n=5)$ for female Long-Evans rats, and $125 \pm 28.1$ $\mu$ mol-min/l (SEM, $n=6)$ for male Sprague-Dawley rats. These values were not significantly different $(p=0.354$, Student's $t$-test for unpaired data).

Hippocampal and aortic sGC activity. Cholinergic innervation of the hippocampus and cerebral cortex of female Long-Evans rats was depleted by intraventricular microinjection of 192-IgG saporin, as described above, and 2 weeks later the aortae and hippocampi from sham-operated and lesioned animals were removed. The preparation of the $100000 \mathrm{~g}$ supernatant fractions of aorta and hippocampus, and enzyme assay conditions for assessment of sGC activity were as described (Bennett et al, 1992). Samples $(0.2 \mathrm{ml})$ contained $2 \mathrm{mM}$ L-cysteine and GT1061 (10 $\mu \mathrm{M}-10 \mathrm{mM})$. Reactions were initiated by the addition of $44-79 \mu \mathrm{g}$ hippocampal supernatant protein or $20-31 \mu$ g aortic supernatant protein and terminated by the addition of $0.8 \mathrm{ml}$ of $50 \mathrm{mM}$ sodium acetate $(\mathrm{pH} 4.0)$ followed by heating at $90^{\circ} \mathrm{C}$ for $3 \mathrm{~min}$. The cGMP was quantitated by radioimmunoassay and the protein content determined using bovine serum albumin as the standard.

Phosphorylation of ERK1/2. To test for in vivo activation of ERK1/2, separate groups of nonlesioned animals were administered GT1061 (1 mg/kg i.p. $(4.5 \mu \mathrm{mol} / \mathrm{kg}))$ or phosphate-buffered saline. At 60 min after drug administration, the brain of each animal was rapidly removed, dissected into regions (hippocampus, frontal cortex, and cerebellum), and homogenized in lysis buffer containing $50 \mathrm{mM}$ TRIS, $\mathrm{pH}$ 7.4, $50 \mathrm{mM}$ sodium pyrophosphate, $1 \mathrm{mM}$ EDTA, $1 \%$ Triton X-100, $1 \mathrm{mM}$ dithiothreitol, $1 \mathrm{mM}$ PMSF, $1 \mathrm{mM} \mathrm{NaF}, 1 \mathrm{mM} \mathrm{NaVO}$ and protease inhibitors (Roche Applied Science). Phosphorylated ERK1/2 (pERK) was determined by ELISA using a commercially available kit (Assay Designs Inc., Ann Arbor, MI). In some experiments, rats were administered the NMDA receptor antagonist, MK801 (1 mg/kg, i.p.), 20 min before GT1061. For in vitro brain slice experiments, rats were anesthetized with halothane, decapitated, and the brain rapidly excised into ice-cold sucrose-substituted Krebs' buffer (342 mM sucrose, $4.8 \mathrm{mM}$ $\mathrm{KCl}, 1.2 \mathrm{mM} \mathrm{KH}_{2} \mathrm{PO}_{4}, 1.3 \mathrm{mM} \mathrm{CaCl}_{2}, 1.2 \mathrm{mM} \mathrm{MgSO}_{4}, 25 \mathrm{mM}$ $\mathrm{NaHCO}_{3}$, and $10 \mathrm{mM}$ glucose, $\mathrm{pH} 7.4$, saturated with $95 \%$ $\mathrm{O}_{2} / 5 \% \mathrm{CO}_{2}$ ). The hippocampus was isolated, $400 \mu \mathrm{m}$ slices were cut with a McIlwain tissue chopper, and a small scalpel blade was used to isolate the CA1 region of each slice. Hippocampal mini-slices containing the CA1 region were 
equilibrated for $3 \mathrm{~h}$ in Krebs' buffer ( $118 \mathrm{mM} \mathrm{NaCl}, 4.8 \mathrm{mM}$ $\mathrm{KCl}, 1.2 \mathrm{mM} \mathrm{KH}_{2} \mathrm{PO}_{4}, 1.3 \mathrm{mM} \mathrm{CaCl}_{2}, 1.2 \mathrm{mM} \mathrm{MgSO}_{4}, 25 \mathrm{mM}$ $\mathrm{NaHCO}_{3}$, and $10 \mathrm{mM}$ glucose, saturated with $95 \% \mathrm{O}_{2} / 5 \%$ $\mathrm{CO}_{2}$ ) at room temperature, and then exposed to drug or vehicle for $30 \mathrm{~min}$. At the end of the incubation, the slices were homogenized in lysis buffer, and the content of pERK determined by ELISA.

Synthesis of GT1061. GT1061, prepared under GMP conditions, was obtained from GB Therapeutics Ltd (Mississauga, ON, Canada). GT1061 was synthesized from 4-methyl-5-(2-hydroxyethyl)-1,3-thiazole by adaptation of standard acidic nitration procedures, followed by salt formation with $\mathrm{HCl}$ and crystallization. Purity as assessed by chromatography and spectroscopy was greater than $98 \%$. GT1061 is a crystalline white solid: $\mathrm{mp} 107-108^{\circ} \mathrm{C}(\mathrm{d})$.

Statistical analyses. In the MWT and the DMTS task, data were evaluated with a repeated measures ANOVA procedure (SPSS 11.0) as previously recommended (Kirk, 1982; Ringo, 1991). Post hoc tests were conducted using Fisher's LSD method. Data from other experiments were analyzed by the appropriate statistical test, as indicated. Differences were considered to be statistically significant if $p<0.05$.

\section{RESULTS}

\section{Histology}

Examination of the acetylcholinesterase stained sections using NIH Image densitometry showed that after bilateral injections of 192 IgG-saporin into the nucleus basalis magnocellularis and medial septum, animals exhibited significantly less acetylcholinesterase staining in both the neocortex and hippocampus as compared to sections obtained from control animals $(t=-12.9, p<0.0001$, and $t=-7.38, p<0.0001$, respectively, Figure 2). Compared to the focal injections of 192 IgG-saporin, we found a similar degree of depletion of acetylcholinesterase in the neocortex and hippocampus after intraventricular administration of 192 IgG-saporin (both $p$ 's $>0.6$ ).

\section{Moving Hidden Platform Morris Water Task}

We conducted separate ANOVAs for donepezil-treated and GT1061-treated groups on latency to find the platform on days when it was in novel locations. There were two within subject factors, day and trial. In the case of donepezil, the ANOVA shows that the groups did differ significantly from one another $(\mathrm{F}(5,46)=2.55, p<0.04)$. The animals improved significantly over days $(\mathrm{F}(5,230)=6.07, p<0.0001)$ and trials $(\mathrm{F}(7,322)=184.67, p<0.0001)$. In the case of GT1061, the ANOVA shows that the groups did differ significantly from one another $(\mathrm{F}(5,48)=3.33, p<0.01)$. The animals improved significantly over days $(F(5,240)=$ 7.52, $p<0.0001)$ and trials $(\mathrm{F}(7,336)=90.3, p<0.0001)$. In both cases, post hoc analysis shows that the sham control group exhibited shorter latencies compared to all other groups (all $p$ 's $<0.0001$ ).

For further illustration of the main trend, Figure 3a shows the average latency to find the hidden platform on trial 2 on days when the platform was in novel locations. Previous
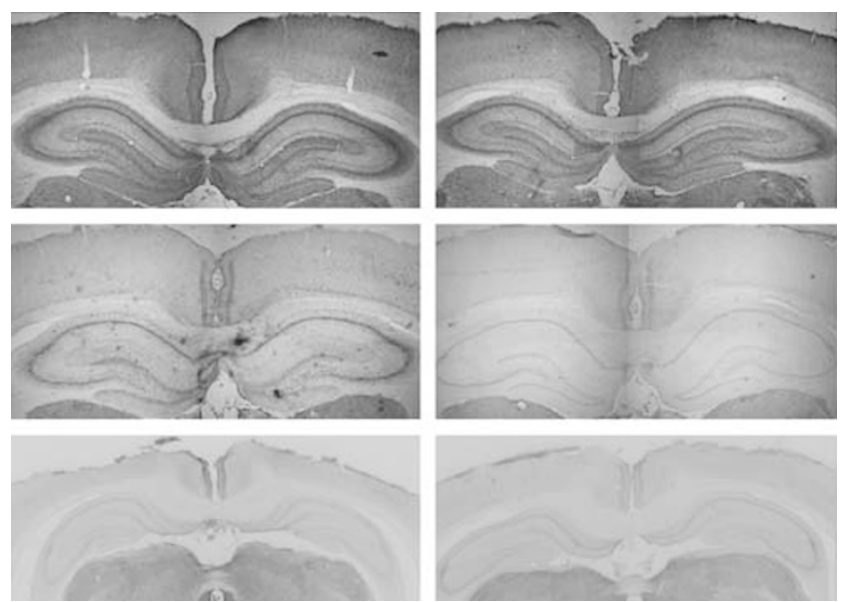

Figure 2 Acetylcholinesterase staining in rat brain after either bilateral injections of 192-lgG saporin into the nucleus basalis magnocellularis and medial septum (middle panels), or intraventricular injections of 192 lgGsaporin (bottom panels). Stained sections from vehicle control (top panels), or 192 lgG-saporin-treated animals were analyzed using $\mathrm{NIH}$ image densitometry. Animals treated with 192 lgG-saporin exhibited significantly less staining in both the cerebral cortex $(t=-12.9, p<0.000 \mathrm{I})$ and hippocampus $(t=-7.38, p<0.000 \mathrm{I})$ compared to sections from the control animals.
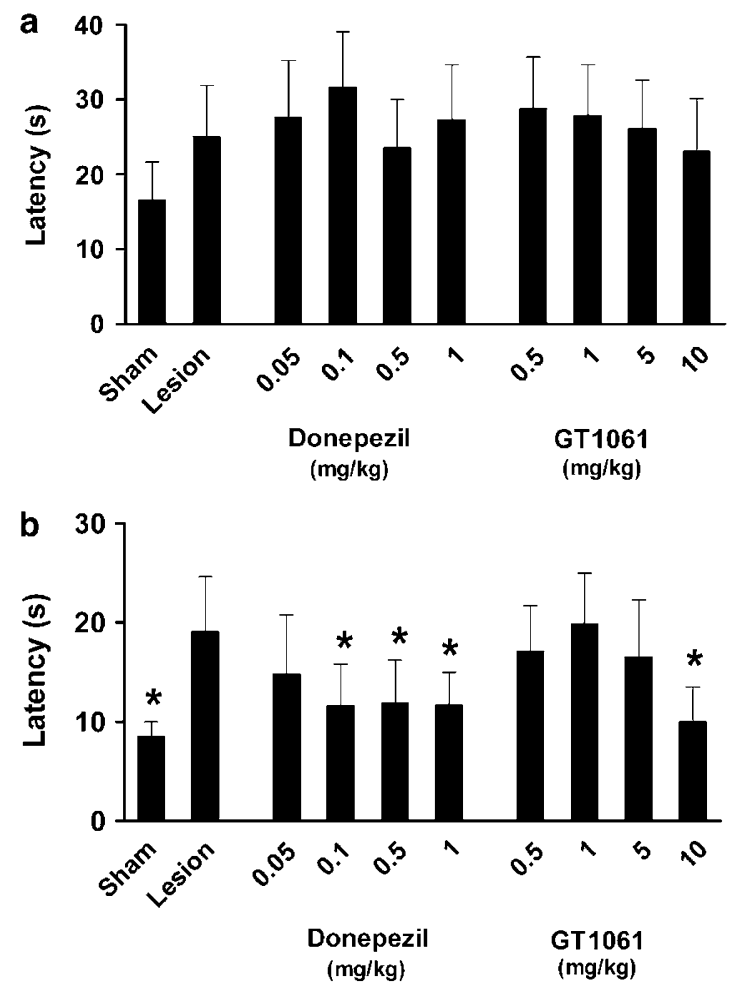

Figure 3 Effect of oral administration of GTI06I $(0.5-10 \mathrm{mg} / \mathrm{kg})$ and donepezil $(0.05-1 \mathrm{mg} / \mathrm{kg})$ in the moving hidden platform Morris water task. (a) The data represents the average time to find the hidden platform on trial 2 on days when the platform was in novel locations. Data are expressed as the mean \pm SEM $(n=10)$. The groups did not differ significantly from one another $(F(9,78)=1.66, p=0.1$ l, repeated measures ANOVA). (b) The data represents the average time to find the hidden platform on trial 2 on days when the platform was in the same location. Data are expressed as the mean \pm SEM $(n=10)$. * $p<0.05$ vs lesion, repeated measures ANOVA $(F(9,78)=2.09, p=0.04)$. 
work in our laboratory has shown that this trial provides a good index of new learning. Trial 1 performance was not different between any of the groups and all of the groups improved by approximately $16 \mathrm{~s}$ between trial 1 and 2 . An ANOVA on the trial 2 data revealed that the treatment groups were not different from one another $(\mathrm{F}(9,78)=1.66$, $p=0.11$ ), indicating that in lesioned animals, neither donepezil nor GT1061 altered the animal's capacity for new learning.

In similar fashion, we analyzed the latency to find the platform on days when the hidden platform remained in the same location as the previous day for groups receiving donepezil. The ANOVA shows that the groups did not differ significantly from one another $(\mathrm{F}(5,46)=0.42$, $p=0.83)$. The groups did improve significantly over days $(\mathrm{F}(5,230)=4.4, \quad p<0.0007)$, and trials $(\mathrm{F}(7,322)=59.4$, $p<0.0001)$. For the groups receiving GT1061 there was a significant difference between the groups $(F(5,48)=2.39$, $p=0.05)$. The groups did improve significantly over days $(\mathrm{F}(5,25)=3.62, p<0.004)$. Again, for simplicity of illustration, Figure $3 \mathrm{~b}$ shows the group data for performance on trial 2 for all days when the platform was in the same location as the previous day. Earlier work has shown that this is a sensitive index of memory performance, which should reveal effects from the previous day's learning and the first trial reminder. The trial 1 performance showed a similar trend to that of trial 2, but we selected trial 2 because of its lower within group variance and hence greater sensitivity. A repeated measures ANOVA examining the group differences on trial 2 only when the platform was in the same locations as the previous day shows that the groups differed significantly from one another $(\mathrm{F}(9,78)=2.09, p=0.04)$. With donepezil, doses of $0.1 \mathrm{mg} /$ $\mathrm{kg}$ or higher produced significant benefit in cholinergicdepleted rats $(p$ 's $<0.01)$. GT1061, at an oral dose of $10 \mathrm{mg} /$ $\mathrm{kg}$, produced the same beneficial effect on memory performance as did donepezil $(p=0.54)$ and produced performance that was not different from sham lesion rats $(p=0.60)$ but superior to depleted control rats $(p<0.002)$. There were no significant differences in swim speeds associated with cholinergic depletions or either drug (all p's $>0.2$ ).

\section{Delayed Matching to Sample Task}

The overall repeated measures ANOVA on percent correct showed statistically significant differences between conditions $(\mathrm{F}(7,63)=25.0, p<0.001)$. Before surgery, rats made $75.0 \%$ correct choices at a 4 -min delay between sample and choice phases and this was significantly decreased to approximately $55.0 \%$ in the same animals after cholinergic depletion $(p<0.0006)$. This deficit in the visual memory task was large and sustained throughout the experiment. All doses of GT1061 significantly improved performance. Indeed, the highest dose of GT1061 restored performance in the DMTS task to a level that was not significantly different from the presurgery level (Figure 4). The two higher doses of donepezil improved performance, but the lowest dose $(0.1 \mathrm{mg} / \mathrm{kg})$ had no effect. In this assay, GT1061 was equipotent (on a per $\mathrm{mg}$ basis) with donepezil at the $1 \mathrm{mg} / \mathrm{kg}$ dose, and at $10 \mathrm{mg} / \mathrm{kg}$, the improvement in performance was significantly greater than that of any of the

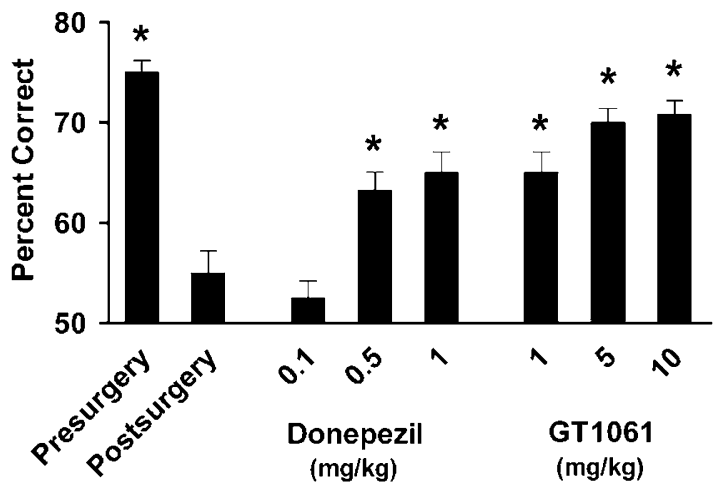

Figure 4 Effect of oral administration of GTI06I (I-10 mg/ $/ \mathrm{kg})$ and donepezil $(0.1-1 \mathrm{mg} / \mathrm{kg})$ in the DMTS task, which measures recognition memory performance. The decreased accuracy in the task after cholinergic depletion was reversed at all doses of GTI06I and at higher doses of donepezil. Data are expressed as the mean $\pm \operatorname{SEM}(n=10)$. ${ }^{*} p<0.01$ vs postsurgery, repeated measures ANOVA $(F(\overline{7}, 63)=25.0, p<0.00 \mathrm{I})$.

doses of donepezil $(p$ 's $<0.01)$. It should be noted that the oral bioavailability of donepezil approaches $100 \%$, whereas that of GT1061 is about $20 \%$ (see below). In the study that assessed whether GT1061 improved performance in nonlesioned animals, rats made $77 \pm 2 \%$ (SEM) correct choices on the trial day before GT1061, and $83.5 \pm 4.9 \%$ correct choices on the day after. Oral administration of $10 \mathrm{mg} / \mathrm{kg}$ GT1061 improved performance to $87.8 \pm 4.3 \%$ correct choices, but the increase was not statistically significant $(\mathrm{F}(2,6)=3.4$, $p=0.161)$.

\section{Pharmacokinetic Studies}

GT1061 was rapidly absorbed after i.p. or oral administration with peak plasma concentrations occurring 3 min after dosing (Figure 5). Calculation of the AUCs indicated an oral bioavailability of $20.5 \pm 11.7 \%$, (range of $9-41.3 \%$ ), and an i.p. bioavailability of $52.8 \pm 10.4 \%$ (range of 36.6 to $64.7 \%$ ). After i.v. administration, values for $V_{\mathrm{d}}(1 / \mathrm{kg}), \mathrm{Cl}(1 / \mathrm{min} / \mathrm{kg})$ and elimination half-life $(\mathrm{min})$ (derived from $V_{\mathrm{d}}$ and $\mathrm{Cl}$ ) were 2.5, 0.079, and 21.6, respectively. For oral administration the values were: 3.4, 0.072, and 32.6, and for i.p. administration the values were: 2.2, 0.08, and 19.0. Calculations for oral and i.p. $V_{\mathrm{d}}$ and $\mathrm{Cl}$ values used the derived bioavailability values for the fractional dose absorbed. In the brain bioavailability study, the mean plasma level of GT1061 $5 \mathrm{~min}$ after oral dosing was $0.52 \pm 0.25 \mathrm{nmol} / \mathrm{ml}$ (SD, $n=6$ ), whereas the mean tissue concentration of GT1061 in hippocampus (expressed as $\mathrm{nmol} / \mathrm{g}$ wet weight, roughly equivalent to $\mathrm{nmol} / \mathrm{ml}$ assuming that $1 \mathrm{~g}$ of tissue has a volume of $1 \mathrm{ml}$ ) was $0.67 \pm 0.42 \mathrm{nmol} /$ g wet weight (SD, $n=6$ ) (Figure 6). Plasma and hippocampal levels of GT1061 were not significantly different from each other (Student's $t$-test for paired data), and in every animal the brain levels of GT1061 were higher than the plasma levels (Figure 6). The ratio of brain to blood GT1061 was $1.3 \pm 0.28$, a value that may underestimate the true brain penetration of GT1061, since during the time between killing and completion of the dissection of the hippocampus (ca. $5 \mathrm{~min}$ ) further metabolic biotransformation of GT1061 in the brain tissue may have occurred. 


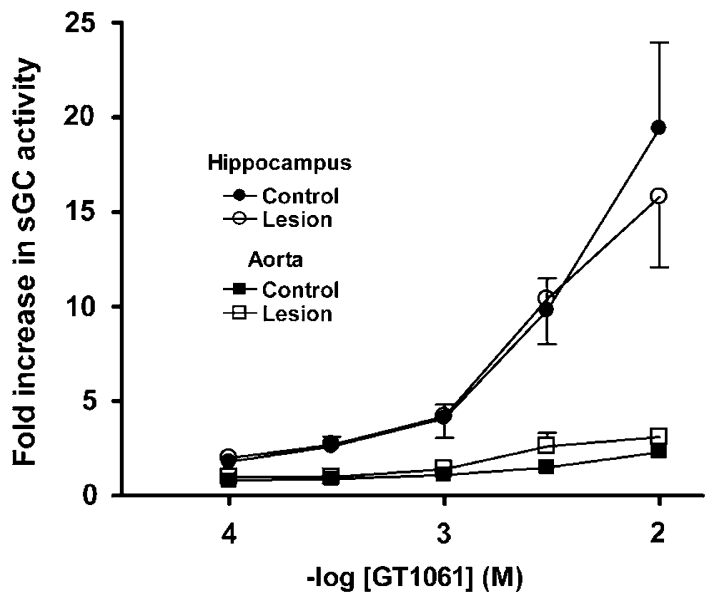

Figure 5 Time course of plasma concentrations of GT I06 I after i.v., i.p., and oral administration of $50 \mu \mathrm{mol} / \mathrm{kg}$ GTI06I. Data are expressed as the mean \pm SEM $(n=5-6)$. The oral bioavailability of GTI06I was $20.5+11.7 \%$.

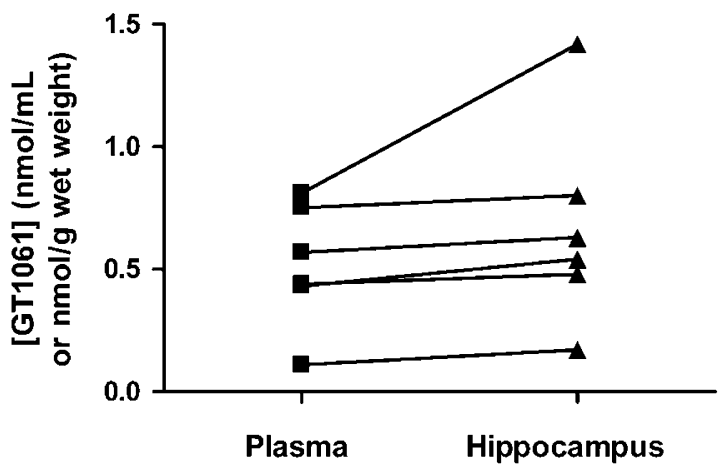

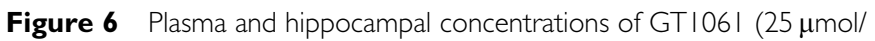
$\mathrm{kg}) 5 \mathrm{~min}$ after oral administration. The mean plasma concentration of GTI06/was $0.52 \pm 0.25 \mathrm{nmol} / \mathrm{ml}(\mathrm{SD}, n=6)$ and the mean tissue concentration of GTI06I in hippocampus was $0.67 \pm 0.42 \mathrm{nmol} / \mathrm{g}$ wet weight (SD, $n=6)$. These values were not significantly different to each other (Student's $t$-test for paired data). The ratio of brain to plasma GTI06I was $1.3 \pm 0.28$.

GT1061 exhibited pharmacokinetic properties similar to classic nitrates such as GTN and isosorbide dinitrate. Absorption was rapid, and bioavailability after oral administration was somewhat variable (9-41\%). The large volume of distribution $(2.2-3.41 / \mathrm{kg}$, depending on route of administration) indicated that the drug was distributed extensively in tissues. This was clearly evident in the brain, where drug concentrations exceeded those in plasma. Extrapolation of the plasma levels of drug obtained in the pharmacokinetic study to the doses of drug that demonstrated efficacy in the behavioral tests suggest that therapeutic concentrations of GT1061 are in the submicromlar range.

\section{Hippocampal and Aortic sGC Activity}

GT1061 caused a concentration-dependent increase in sGC activity in both aortic and hippocampal homogenates (Figure 7), although the degree of activation in the hippocampus was markedly greater. Enzyme activation by GT1061 in hippocampal homogenates from control and

\section{Phosphorylation of ERK1/2}

In the in vivo assessment of phosphorylated ERK1/2 (pERK), GT1061 increased the levels of pERK in the cerebral cortex, but had no effect in the cerebellum (Figure 8a). In the hippocampus, pERK levels were increased by GT1061, but the increase did not reach statistical significance. However, when GT1061 was administered to animals that had been pretreated with the NMDA receptor antagonist, MK-801, the increase in hippocampal pERK caused by GT1061 was significant (Figure 8a, inset), suggesting that in the hippocampus, the effects of GT1061 are more evident in circumstances where input from upstream effectors has been compromised. Qualitatively, this is consistent with the lack of a significant effect of GT1061 on cognition in unlesioned animals. We also compared the effect of GT1061 to that of the cholinergic muscarinic receptor agonist, carbachol, in hippocampal mini-slices containing the CA1 region (Figure 8b). Both drugs significantly increased the amount of pERK in this in vitro preparation. 
a
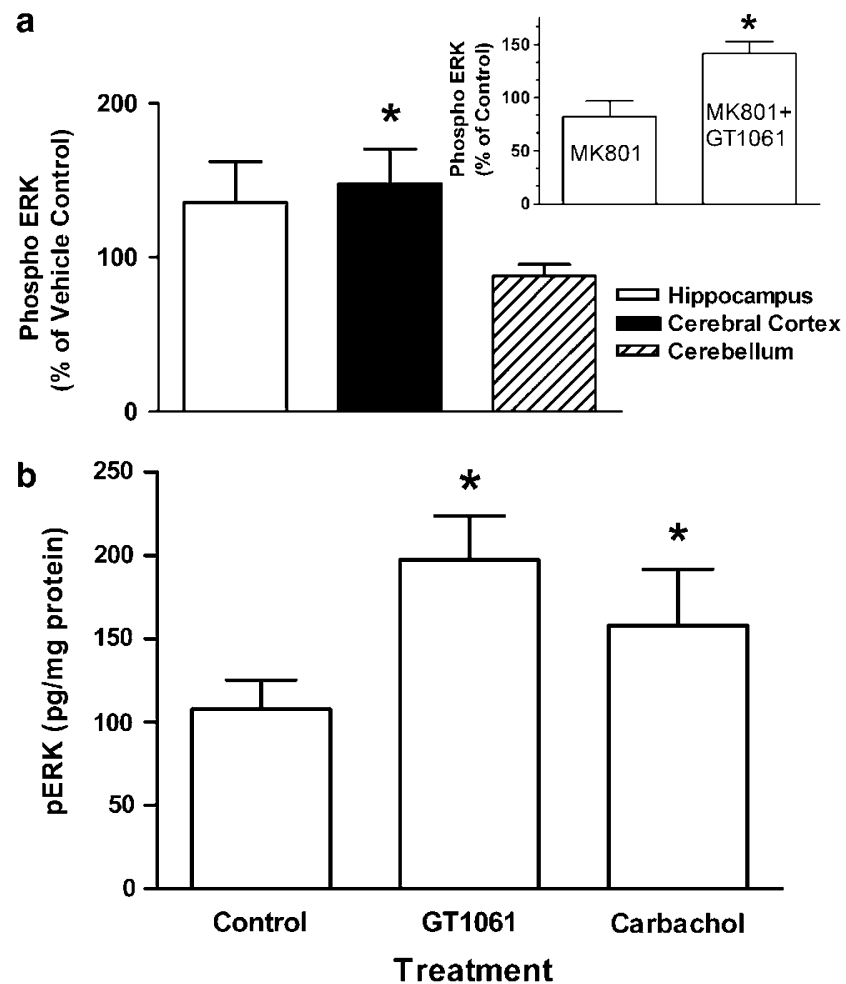

Figure 8 Effect of GTI06I on ERK phosphorylation. (a) Levels of pERKI/2 in different brain regions 60 min after a single i.p. injection of GTI06 I (I mg/ $/ \mathrm{kg})$. Data are expressed as a percentage of the levels of pERK measured in saline-injected animals (mean \pm SEM, $n=6$ ). * $p<0.05$ vs control, Student's t-test for unpaired data. Inset: Levels of pERK in the hippocampus $60 \mathrm{~min}$ after a single i.p. injection of GTI06। (I mg/ $/ \mathrm{kg}$ ) with or without prior treatment of animals with the NMDA receptor antagonist, MK-80 I ( I mg/kg, i.p.). Data are expressed as a percentage of the levels of pERK measured in saline-injected animals (mean \pm SEM, $n=6$ ). $* p<0.05$ vs control, Student's $t$-test for unpaired data. (b) Levels of pERKI/2 in the CAI region of hippocampal mini-slices in vitro after a 30 min incubation with GTI06I (50 $\mu \mathrm{M})$ or the cholinergic muscarinic receptor agonist, carbachol $(50 \mu \mathrm{M})$. Both compounds induced an equivalent increase in the content of pERK. Data are expressed as the mean $\pm \operatorname{SEM}(n=5) . * 2<0.05$ vs control, repeated measures ANOVA $(F(2,8)=9.62, p=0.0074$. Pair wise comparisons were performed using Newman-Keuls post hoc test.

\section{DISCUSSION}

Development of pharmacologically effective treatments that alleviate cognitive disorders is a central objective in neuroscience. The high incidence of dementia, in particular $\mathrm{AD}$, in the aging population provides strong impetus to search for new compounds with the potential to reverse the deficits in short-term memory. The neuropathological changes underlying age-related cognitive decline and $\mathrm{AD}$ are complex and poorly understood. However, the loss of forebrain cholinergic neurons that project to the cerebral cortex and hippocampus is a hallmark of this disease. In addition, the deposition of $\mathrm{A} \beta$ in both the brain parenchyma and cerebral blood vessels, and the formation of intracellular neurofibrillary tangles containing tau-protein are also observed. Studies in Alzheimer's patients have revealed that the extent of cholinergic depletion correlates with the severity of memory deficits (Mesulam, 2004). Thus, animal models of cortical and hippocampal cholinergic depletion have been widely used to induce relevant cognitive deficits for the purpose of identifying potential new therapeutic compounds for the treatment of $\mathrm{AD}$.

It is well established that dysfunction in the hippocampus causes spatial learning deficits in tasks such as the MWT (Redish and Touretzky, 1998; Silva et al, 1998a) However, the role of the hippocampus in visual memory and recognition tasks has been controversial. It was recently confirmed that the hippocampus is essential for nonspatial visual recognition memory in the rat (Prusky et al, 2004). The observation of similarities between cognitive processing in the rat and human is important, but the development of a reliable rat model of recognition memory deficit also provides a powerful tool for development of drugs for $\mathrm{AD}$ and neurodegenerative diseases. It is noteworthy that visual association tests are reported to detect, with high specificity, a substantial proportion of AD patients up to a year before diagnosis (Lindeboom et al, 2002). This paper demonstrates that novel nitrates are a new class of cognition enhancing agents, and also validates the use of the rat DMTS model by assay of the $\mathrm{AD}$ drug donepezil. In the original DMTS work (Prusky et al, 2004), the perirhinal cortex was lesioned by aspiration and the hippocampus lesioned by direct injection of excitotoxic doses of NMDA. We show herein that intraventricular infusion of 192 IgGsaporin induces a reliable cholinergic lesion resulting in visual recognition memory impairment, which is reversed by treatment with GT1061 and donepezil. We note here that with the present results we cannot determine the extent to which damage to noncholinergic cells may contribute to the memory deficit.

Our observation that a novel nitrate, NO mimetic compound exerts beneficial effects in multiple learning paradigms, supports the idea that activation of the sGC/ cGMP signal transduction cascade enhances synaptic efficacy in brain regions responsible for the acquisition, storage, and retention of new learning. Activation of $\mathrm{NO} /$ cGMP signaling represents a new drug target for $\mathrm{AD}$, a disease characterized by generalized, progressive decline in cognitive function due to diffuse neuronal loss and synaptic failure in the hippocampus and cerebral cortex (Scheff and Price, 2003; Selkoe, 2002).

Acetylcholine (ACh), a major modulatory neurotransmitter in the brain, is critically involved in learning and memory. In the cortex and hippocampus, the procognitive actions of ACh are mediated via muscarinic receptors whose activity is primarily excitatory, and involves multiple different ionic conductances. In the hippocampus, cholinergic muscarinic receptor activation leads to increased tissue levels of cGMP (Tonnaer et al, 1991). Importantly, the inhibition of the slow afterhyperpolarizing current (a calcium-activated potassium conductance that underlies spike-frequency adaptation) induced by muscarinic receptor activation in hippocampal CA1 pyramidal neurons can be blocked by inhibitors of sGC and PKG (Krause and Pedarzani, 2000). These data suggest that the neuromodulatory effects of ACh in the brain involve, at least in part, the $\mathrm{NO} / \mathrm{sGC/cGMP}$ signal transduction cascade.

Behavioral studies have demonstrated that NMDA receptors also play an important role in both spatial working memory and long-term memory processes. Blockade of NMDA receptors, or inhibition of NOS activity, impairs performance in the Y-maze test, a model of spatial 
working memory (Yamada et al, 1996). The impairment induced by NMDA receptor blockade could be reversed by intracerebroventricular administration of L-arginine, a nitrosothiol NO donor, or a cGMP analog. Taken together, these studies suggest that both $\mathrm{ACh}$ and glutamate activate receptor systems coupled to the NO/sGC/cGMP signal transduction cascade and that this biochemical cascade is important for synaptic plasticity and the formation of memory.

In previous studies, we found that the novel nitrate, GT715, was less potent than GTN for relaxation of vascular smooth muscle and for decreasing mean arterial pressure, and more effective than GTN for activating sGC in the hippocampus. In simile with these data, the activation of sGC in broken cell preparations of aorta and hippocampus revealed that GT1061 selectively increased enzyme activity in the hippocampus (16- to 20-fold in hippocampus vs 2- to 3 -fold in aorta), confirming that the structure of the nitrate NO mimetic influences tissue selectivity for sGC activation. Additionally, sGC activity was not altered in tissue homogenates from lesioned animals, indicating that cGMP signaling remained intact after depletion of cholinergic innervation of the hippocampus. Soluble GC is a heterodimer of $\alpha$ and $\beta$ subunits, and recent studies of the distribution of $\alpha_{1}$ and $\alpha_{2}$ subunits of sGC have shown that the $\alpha_{2}$ subunit is expressed in relatively high abundance in brain, especially in the hippocampus, where the $\alpha_{2} \beta_{1}$ rather than the $\alpha_{1} \beta_{1}$ heterodimer of sGC is the major isoform present (Mergia et al, 2003). Furthermore, the $\alpha_{2}$ subunit interacts with a PDZ domain of PSD95 (Russwurm et al, 2001). As this scaffolding protein couples the NMDA receptor with neuronal NOS, this suggests an important role for the $\alpha_{2} \beta_{1}$ heterodimer isoform in synaptic transmission. The enhanced activation of hippocampal sGC by GT1061 suggests that there is selectivity for targeting the $\alpha_{2} \beta_{1}$ isoform of sGC.

A number of kinase signaling cascades may be involved in signal transduction by NO/cGMP resulting in enhanced cognition and memory; chief among these being the ERK cascade ( $\mathrm{Lu}$ et al, 1999). Activation of ERK1/2 is believed to play a critical role in cognition and memory mediated through phosphorylation and activation of CREB, and may serve as a point of convergence for a number of upstream signaling molecules, including glutamate acting via NMDA receptors and ACh acting via cholinergic muscarinic and nicotinic receptors (Sweatt, 2004). Activation of transcription regulating factors, such as CREB, leading to protein synthesis is a requirement for the consolidation of new learning into long-term memory (Silva et al, 1998b). ERK1/2 activation can be mediated via the protein kinases, PKA or PKC, whereas CREB can be activated and phosphorylated via CaMK IV, PKA, and RSK2, the last mediating the activation of CREB by the ERK cascade (Xing et al, 1996). Studies suggest that PKG provides a parallel pathway to PKA-signaling in both phases of LTP, with PKG and PKA pathways performing complementary roles (Lu et al, 1999). NO/cGMP and PKG contribute to CREB phosphorylation, mediated by the ERK cascade, and also possibly in part via the CaMK pathway ( $\mathrm{Lu}$ et al, 1999). Interestingly, experiments with YC-1, an agent that augments sGC activation, produced an enhancement of LTP in rat hippocampus and amygdala via an $\mathrm{NO} / \mathrm{cGMP} / \mathrm{PKG} / \mathrm{ERK}$ pathway culminating in CREB phosphorylation (Chien et al, 2003). In in vitro brain slice experiments, we found that GT1061 increased the levels of $\mathrm{pERK}$ in the CA1 region of the hippocampus. In addition, the finding that in vivo administration of GT1061, at doses associated with cognitive improvement, increased ERK1/2 phosphorylation in the hippocampus and cerebral cortex, provides evidence for a mechanism of GT1061 cognition enhancement via sGC/cGMP signaling mediated at least in part by the ERK cascade.

Arancio and co-workers reported recently that activation of $s G C$ or addition of a cGMP analog were able to protect against the $\mathrm{A} \beta$-induced impairment of hippocampal CA1 region LTP (Puzzo et al, 2005). In mouse hippocampal slices treated with agents in the presence of $\mathrm{A} \beta_{1-42}$ before induction of LTP , A $\beta$ inhibited LTP and blocked elevation of pCREB in response to tetanus; the activation of sGC had no effect on basal LTP and pCREB levels in the absence of the tetanus stimulation. A further study provided a pathway for $\mathrm{A} \beta$-induced disruption of NO/cGMP signaling initiated by binding of $\mathrm{A} \beta$ to the $\alpha 7$ nicotinic $\mathrm{ACh}$ receptor (Snyder et al, 2005). Treatment of neurons with $\mathrm{A} \beta$ was reported to produce a depression of NMDA-evoked currents and reduced CREB phosphorylation via a mechanism involving dissociation of the synaptic protein, PSD95, from the NMDA receptor. It is reasonable to assume that this event will seriously disrupt NO/cGMP signaling, since both sGC and nNOS are known to be bound to NMDA receptorassociated PSD95 (Brenman and Bredt, 1997; Mergia et al, 2003; Russwurm et al, 2001). Thus, evidence is growing justifying intervention with $\mathrm{NO}$ mimetic drugs in treatment of AD.

In summary, we have shown for the first time that the visual recognition memory deficit induced by cholinergic depletion in a rat model can be fully reversed by drug administration. All current clinical agents approved for use in mild to moderate $\mathrm{AD}$ attempt to salvage the residual functionality of damaged and degenerating cholinergic neurons, whereas we demonstrate herein an alternative approach whereby the function of the signaling apparatus is modulated to compensate for damage to the cholinergic circuitry. The drug that supports this postulate is a novel nitrate NO mimetic, GT1061. Importantly, this compound displays significant oral bioavailability and brain penetration, and efficacy that, in the visual memory recognition DMTS task, was superior to the clinical AD drug, donepezil. Moreover, GT1061 reverses the cognitive deficits induced by depletion of forebrain ACh in two different learning paradigms, reinforcing both the previous observations with the novel nitrate, GT715, and the potential for development of nitrate NO mimetics as new therapeutic agents. GT1061 induces a selective activation of sGC in the hippocampus compared to the vasculature, predicting that the beneficial CNS actions of the drug will occur at lower plasma concentrations than will any potential undesirable hypotensive actions. GT1061 was observed to activate a pathway in the hippocampus, the ERK cascade, (a) known to be modulated by sGC/cGMP signaling, and (b) which represents a necessary pathway in the formation of long-term memory. Novel nitrates, as represented by GT1061, are a class of compounds that can be exploited in the discovery and development of new therapeutic agents for the treatment of neurodegenerative diseases such as Alzheimer's disease. 


\section{ACKNOWLEDGEMENTS}

We thank Dr Khem Jhamandas for helpful discussions and critical reading of the manuscript. Mrs Diane Anderson, Ms Margo Poklewska-Koziel, and Neuroinvestigations Inc., Lethbridge, Alberta are thanked for technical assistance. Financial support for this project was provided by GB Therapeutics Ltd, Mississauga Ontario, The Institute for the Study of Aging (to GRJT), and The Heart and Stroke Foundation of Ontario (Grant T-5162 to BMB). RJS is an Alberta Heritage Medical Scientist.

\section{REFERENCES}

Areosa SA, Sherriff F, McShane R (2005). Memantine for dementia. Cochrane Database Syst Rev 3: CD003154.

Artz JD, Toader V, Zavorin SI, Bennett BM, Thatcher GR (2001). In vitro activation of soluble guanylyl cyclase and nitric oxide release: a comparison of NO donors and NO mimetics. Biochemistry 40: 9256-9264.

Bartus RT, Dean III RL, Beer B, Lippa AS (1982). The cholinergic hypothesis of geriatric memory dysfunction. Science 217: 408-414.

Bennett BM, McDonald BJ, Nigam R, Simon WC (1994). Biotransformation of organic nitrates and vascular smooth muscle cell function. Trends Pharmacol Sci 15: 245-249.

Bennett BM, McDonald BJ, St James MJ (1992). Hepatic cytochrome P-450-mediated activation of rat aortic guanylyl cyclase by glyceryl trinitrate. J Pharmacol Exp Ther 261: 716-723.

Bernabeu R, Schroder N, Quevedo J, Cammarota M, Izquierdo I, Medina JH (1997). Further evidence for the involvement of a hippocampal cGMP/cGMP-dependent protein kinase cascade in memory consolidation. Neuroreport 8: 2221-2224.

Bonkale WL, Winblad B, Ravid R, Cowburn RF (1995). Reduced nitric oxide responsive soluble guanylyl cyclase activity in the superior temporal cortex of patients with Alzheimer's disease. Neurosci Lett 187: 5-8.

Brenman JE, Bredt DS (1997). Synaptic signaling by nitric oxide. Curr Opin Neurobiol 7: 374-378.

Chien WL, Liang KC, Teng CM, Kuo SC, Lee FY, Fu WM (2003). Enhancement of long-term potentiation by a potent nitric oxideguanylyl cyclase activator, 3-(5-hydroxymethyl-2-furyl)-1-benzyl-indazole. Mol Pharmacol 63: 1322-1328.

Courtney C, Farrell D, Gray R, Hills R, Lynch L, Sellwood E et al (2004). Long-term donepezil treatment in 565 patients with Alzheimer's disease (AD2000): randomised double-blind trial. Lancet 363: 2105-2115.

Karnovsky MJ, Roots L (1964). A 'direct-coloring' thiocholine method for cholinesterases. J Histochem Cytochem 12: 219-221.

Kirk RE (1982). Experimental Design, 2nd edn. Wadsworth: Belmont CA.

Krause M, Pedarzani P (2000). A protein phosphatase is involved in the cholinergic suppression of the $\mathrm{Ca}(2+)$-activated $\mathrm{K}(+)$ current sI(AHP) in hippocampal pyramidal neurons. Neuropharmacology 39: 1274-1283.

Lindeboom J, Schmand B, Tulner L, Walstra G, Jonker C (2002). Visual association test to detect early dementia of the Alzheimer type. J Neurol Neurosurg Psychiatry 73: 126-133.

Lu YF, Kandel ER, Hawkins RD (1999). Nitric oxide signaling contributes to late-phase LTP and CREB phosphorylation in the hippocampus. J Neurosci 19: 10250-10261.

Mergia E, Russwurm M, Zoidl G, Koesling D (2003). Major occurrence of the new alpha2betal isoform of NO-sensitive guanylyl cyclase in brain. Cell Signal 15: 189-195.

Mesulam M (2004). The cholinergic lesion of Alzheimer's disease: pivotal factor or side show? Learn Mem 11: 43-49.

Pizzo DP, Waite JJ, Thal LJ, Winkler J (1999). Intraparenchymal infusions of 192 IgG-saporin: development of a method for selective and discrete lesioning of cholinergic basal forebrain nuclei. J Neurosci Methods 91: 9-19.

Prickaerts J, de Vente J, Honig W, Steinbusch HW, Blokland A (2002). cGMP, but not cAMP, in rat hippocampus is involved in early stages of object memory consolidation. Eur J Pharmacol 436: $83-87$.

Prickaerts J, Steinbusch HW, Smits JF, de Vente J (1997). Possible role of nitric oxide-cyclic GMP pathway in object recognition memory: effects of 7-nitroindazole and zaprinast. Eur $J$ Pharmacol 337: 125-136.

Prusky GT, Douglas RM, Nelson L, Shabanpoor A, Sutherland RJ (2004). Visual memory task for rats reveals an essential role for hippocampus and perirhinal cortex. Proc Natl Acad Sci USA 101: 5064-5068.

Puzzo D, Vitolo O, Trinchese F, Jacob JP, Palmeri A, Arancio O (2005). Amyloid-beta peptide inhibits activation of the nitric oxide/cGMP/cAMP-responsive element-binding protein pathway during hippocampal synaptic plasticity. $J$ Neurosci 25: 6887-6897.

Redish AD, Touretzky DS (1998). The role of the hippocampus in solving the Morris water maze. Neural Comput 10: 73-111.

Reynolds JN, Bennett BM, Boegman RJ, Jhamandas K, Ratz JD, Zavorin SI et al (2002). Neuroprotection against ischemic brain injury conferred by a novel nitrate ester. Bioorg Med Chem Lett 12: $2863-2866$.

Ringo JL (1991). Memory decays at the same rate in macaques with and without brain lesions when expressed in d' or arcsine terms. Behav Brain Res 42: 123-134.

Russwurm M, Wittau N, Koesling D (2001). Guanylyl cyclase/PSD95 interaction: targeting of the nitric oxide-sensitive alpha2beta1 guanylyl cyclase to synaptic membranes. J Biol Chem 276: 44647-44752.

Scheff SW, Price DA (2003). Synaptic pathology in Alzheimer's disease: a review of ultrastructural studies. Neurobiol Aging 24: $1029-1046$.

Selkoe DJ (2002). Alzheimer's disease is a synaptic failure. Science 298: 789-791.

Silva AJ, Giese KP, Fedorov NB, Frankland PW, Kogan JH (1998a). Molecular, cellular, and neuroanatomical substrates of place learning. Neurobiol Learn Mem 70: 44-61.

Silva AJ, Kogan JH, Frankland PW, Kida S (1998b). CREB and memory. Annu Rev Neurosci 21: 127-148.

Smith S, Dringenberg HC, Bennett BM, Thatcher GR, Reynolds JN (2000). A novel nitrate ester reverses the cognitive impairment caused by scopolamine in the Morris water maze. Neuroreport 11: 3883-3886.

Snyder EM, Nong Y, Almeida CG, Paul S, Moran T, Choi EY et al (2005). Regulation of NMDA receptor trafficking by amyloidbeta. Nat Neurosci 8: 1051-1058.

Sweatt JD (2004). Mitogen-activated protein kinases in synaptic plasticity and memory. Curr Opin Neurobiol 14: 311-317.

Thatcher GR, Nicolescu AC, Bennett BM, Toader V (2004). Nitrates and NO release: contemporary aspects in biological and medicinal chemistry. Free Radic Biol Med 37: 1122-1143.

Tonnaer JA, Cheung CL, De Boer T (1991). cGMP formation and phosphoinositide turnover in rat brain slices are mediated by pharmacologically distinct muscarinic acetylcholine receptors. Eur J Pharmacol 207: 183-188.

Xing J, Ginty DD, Greenberg ME (1996). Coupling of the RASMAPK pathway to gene activation by RSK2, a growth factorregulated CREB kinase. Science 273: 959-963.

Yamada K, Noda Y, Hasegawa T, Komori Y, Nikai T, Sugihara H et al (1996). The role of nitric oxide in dizocilpine-induced impairment of spontaneous alternation behavior in mice. J Pharmacol Exp Ther 276: 460-466.

Zavorin SI, Artz JD, Dumitrascu A, Nicolescu A, Scutaru D, Smith SV et al (2001). Nitrate esters as nitric oxide donors: SS-nitrates. Org Lett 3: 1113-1116. 